\title{
A Case of Recurrent Palpitations in an Elderly Woman with Breast Cancer: Management of Recalcitrant Svt in the Emergency Department
}

Tsang Denise Ann MBBS ${ }^{1}$, Lateef Fatimah FRCS (A\&E), MBBS, FAMS (Em Med) ${ }^{2}$

${ }^{1}$ Medical Officer, Dept of Internal Medicine, Singapore General Hospital.

${ }^{2}$ Senior Consultant, Dept of Emergency Medicine, Singapore General Hospital.

*Corresponding Author: Lateef Fatimah, Senior Consultant, Dept of Emergency Medicine, Singapore General Hospital.

Received date: February 06, 2021; Accepted date: April 06, 2021; Published date: April 09, 2021

Citation: Tsang D Ann, L Fatimah. (2021) A Case of Recurrent Palpitations in an Elderly Woman with Breast Cancer: Management of

Recalcitrant Svt in the Emergency Department. International Journal of Clinical Case Reports and Reviews. 7(1); DOI:10.31579/2690-4861/115

Copyright: (c) 2021 Lateef Fatimah, This is an open-access article distributed under the terms of the Creative Commons Attribution License, which permits unrestricted use, distribution, and reproduction in any medium, provided the original author and source are credited

\begin{abstract}
Supraventricular tachycardia encompasses a group of cardiac rhythm disturbances characterised by a rapid heart rate, which arise from impulses originating above the bundle of His. This condition affects patients across all age groups and is a common occurrence in the emergency setting. We present the case of a 69year-old woman with a history of breast carcinoma, hypertension and hyperlipidaemia, who presented to the emergency department with recurrent palpitations which were confirmed on electrocardiogram to be the result of supraventricular tachycardia. The management of recalcitrant supraventricular tachycardia in the emergency setting, including techniques described in recent literature, as well as special considerations for the elderly and oncological patients in the acute setting will be discussed.
\end{abstract}

Key words: supraventricular tachycardia; left bundle branch block; adenosine; AVNRT

\section{Introduction}

Supraventricular tachycardia (SVT) refers to cardiac rhythm disturbances characterised by a rapid heart rate (greater than 100 beats per minute), attributed to electrical impulses which originate from and are propagated by atrial or atrioventricular nodal tissue proximal to the bundle of His. This condition arises due to re-entry phenomena or automaticity at or above the atrioventricular node, which results in a rapid ventricular response when impulses are transmitted down the bundle of His [1]. By definition, SVT encompasses a variety of tachyarrhythmias arising above the bundle of His. In practice, however, SVT commonly refers to atrioventricular nodal re-entry tachycardia (AVNRT), atrioventricular reentry tachycardia (AVRT) and atrial tachycardia. SVT is a relatively common occurrence in the emergency department. This article will provide an overview of the management of recalcitrant SVT in the emergency setting, including various techniques described in recent literature, as well as the effects of age and malignancy on the acute management of SVT.

\section{Case report}

A 69-year-old woman with a history of metastatic spindle cell carcinoma of the breast, hypertension and hyperlipidaemia presented to the emergency department with sudden-onset palpitations, associated with lightheadedness, breathlessness and slight chest discomfort. She denied any previous episodes of palpitations, recent caffeine or alcohol intake and had no history of cardiac or thyroid disease. Her last chemotherapy session was 10 days ago. On examination, she was afebrile and had a blood pressure of 100/68 mmHg. Her respiratory rate was 19 breaths per minute with $99 \%$ oxygen saturation on room air. Her heart rhythm was regular with no murmurs or gallop. Her jugular venous pressure was normal and there was no lower limb oedema. Auscultation of her lungs, however, revealed decreased air entry on the left. A 12-lead electrocardiogram (ECG) performed in the emergency department demonstrated regular, narrow-complex tachycardia at a rate of 188 beats per minute with absent $\mathrm{P}$ waves, consistent with a diagnosis of SVT (Fig 1A). Vagal manoeuvres were unsuccessful and she was given a rapid intravenous bolus consisting of $6 \mathrm{mg}$ adenosine, which resulted in conversion to sinus rhythm with a left bundle branch block (LBBB) pattern (Fig 1B). Her symptoms recurred 30 minutes later and her ECG rhythm strip confirmed a recurrence of SVT, necessitating a repeat dose of adenosine, administered as a $12 \mathrm{mg}$ intravenous bolus. The second dose of adenosine aborted the arrhythmia, however, the patient again complained of palpitations shortly after. As she remained haemodynamically stable, a decision was made for an infusion of diltiazem, following which, there was conversion to sinus rhythm with the same LBBB pattern. 2 hours later, her ECG showed another recurrence of SVT and she was given a repeat infusion of diltiazem. Review of the patient's serum biochemistry showed potassium of $3.5 \mathrm{mmol} / 1$ (reference 
3.6-5mmol/1), magnesium of $0.75 \mathrm{mmol} / \mathrm{l}$ (reference $0.74-0.97 \mathrm{mmol} / \mathrm{l}$ ) and calcium of $2.03 \mathrm{mmol} / \mathrm{l}$ (reference $2.09-2.46 \mathrm{mmol} / \mathrm{l}$ ). Her full blood count showed a white blood cell count of $6.27 \times 109 / \mathrm{L}$ (reference 4.0-10.0 x 109/L), platelets of 288 x 109/L (reference 140-440 x109/L), haematocrit of $35 \%$ (reference 36-46\%) and haemoglobin of $11.6 \mathrm{~g} / \mathrm{dl}$ (reference $12.0-16.0 \mathrm{~g} / \mathrm{dl}$ ). A chest X-ray was performed which revealed the presence of a moderately-sized left-sided pleural effusion. Thereafter, the patient was admitted for further management. She was subsequently found to have developed a left lung collapse with pleural effusion, secondary to tumour invasion of the left main bronchus, for which she underwent rigid bronchoscopy and stenting. Evaluation of her cardiac telemetry revealed features of pre-excitation consistent with a WolffParkinson-White ECG pattern. Electrophysiologic studies demonstrated orthodromic AVRT and ablation of the right free wall accessory pathway was performed successfully with no complications.

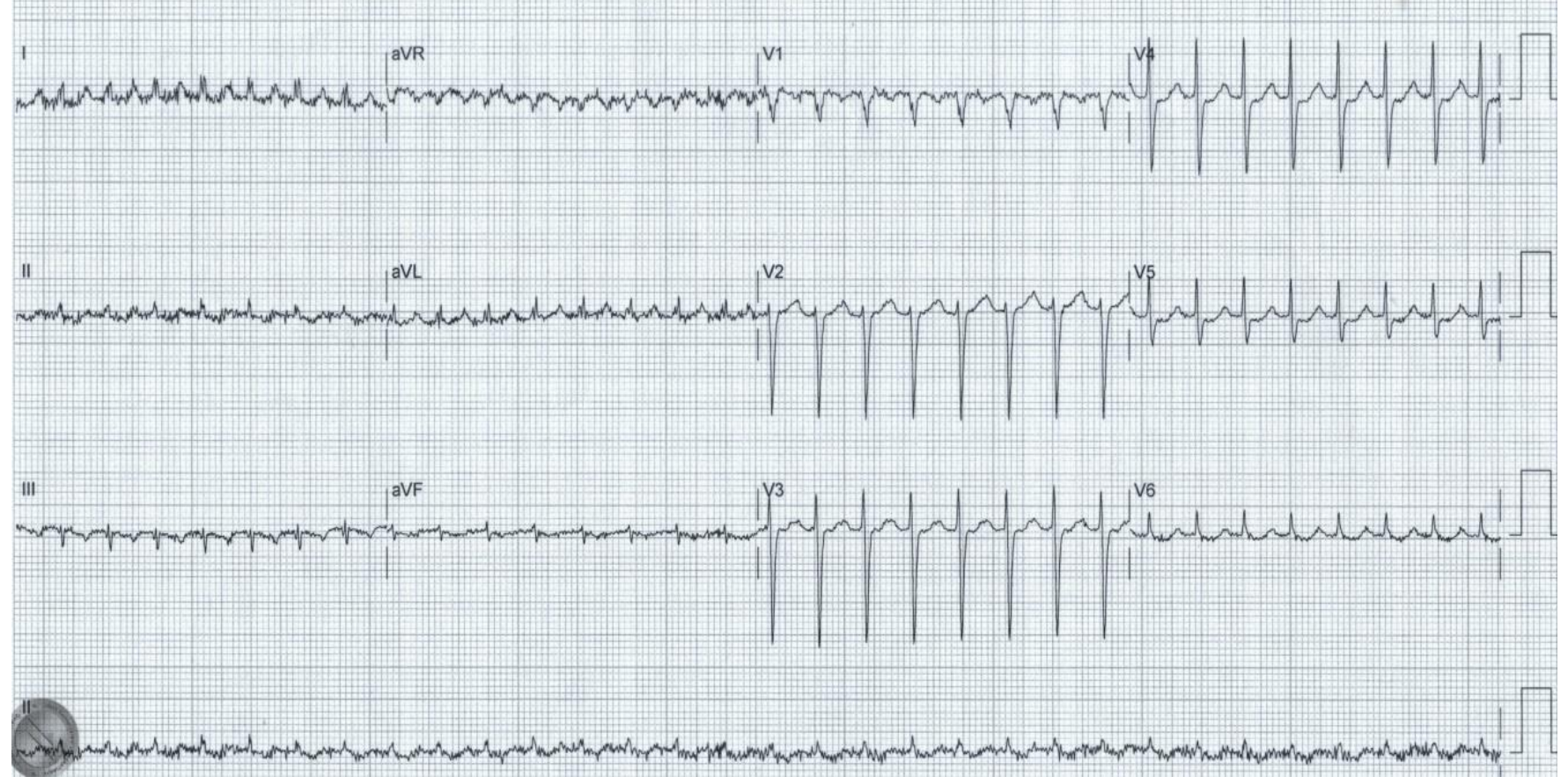

Figure 1A: ECG on arrival at the emergency department showing regular narrow-complex tachycardia at a rate of 188 beats per minute, consistent with a diagnosis of supraventricular tachycardia

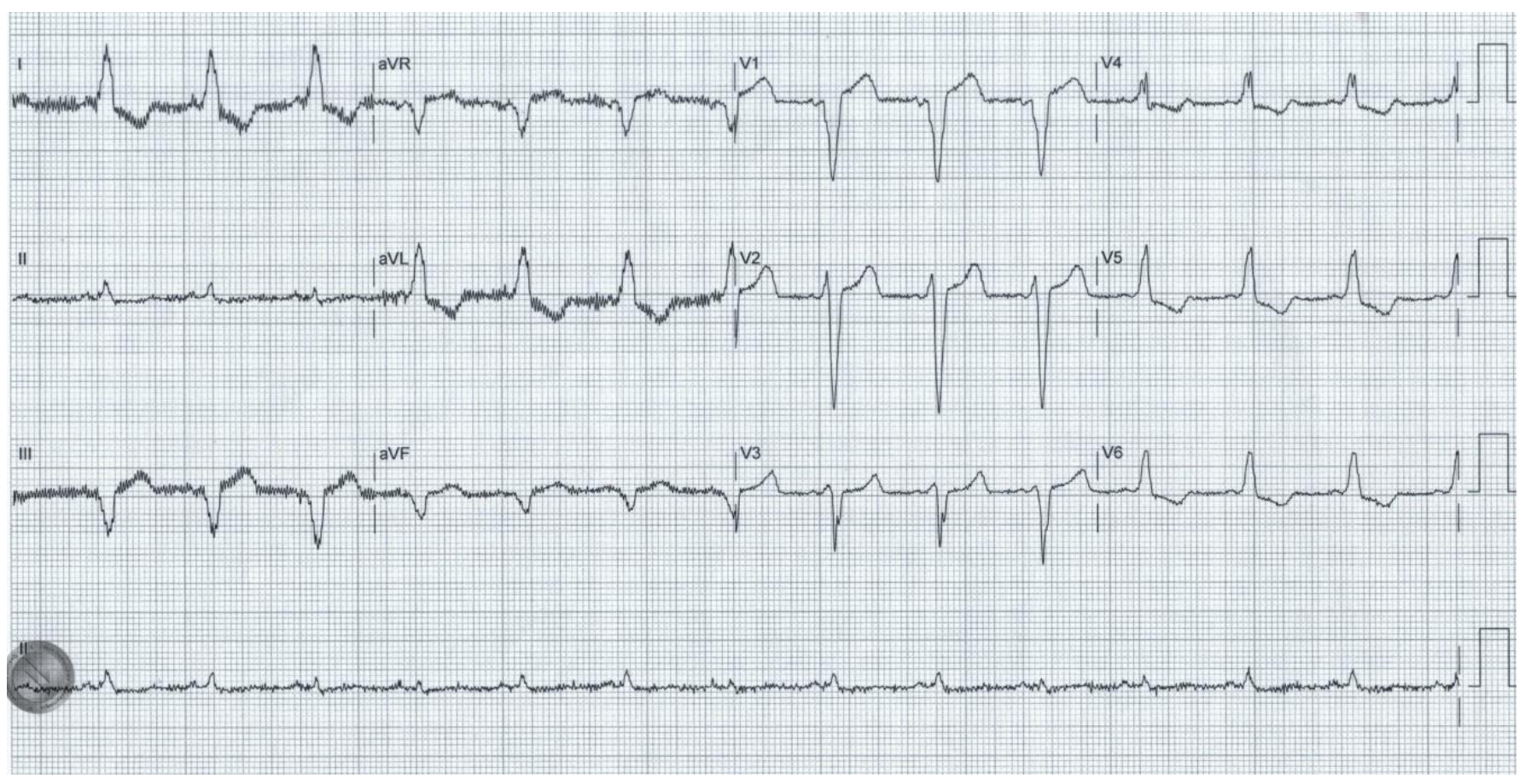

Figure 1B: ECG after administration of $6 \mathrm{mg}$ adenosine showing conversion to sinus rhythm with a left bundle branch block pattern 


\section{Discussion}

Management of recalcitrant supraventricular tachycardia in the emergency department:

Current practice

The first step in the management of SVT entails an assessment of the patient's vital signs. Haemodynamically unstable patients with SVT should undergo immediate direct current (DC) cardioversion beginning at 50J, as per Advanced Cardiac Life Support (ACLS) guidelines. Otherwise, attempts may be made to abort the tachyarrhythmia with vagal manoeuvres. Various techniques have been described in the literature, such as carotid massage, bearing down, coughing, gagging and even immersing the face in cold water [2]. Adenosine is the drug of choice if vagal manoeuvres fail to terminate SVT and should be administered as a rapid intravenous bolus with an initial dose of $6 \mathrm{mg}$. A repeat dose at 12 $\mathrm{mg}$ of adenosine may be administered should the tachycardia persist. Adenosine has a negative chronotropic effect on the heart as it prolongs atrioventricular nodal conduction. Its rapid onset of action and short halflife of less than 10s makes it an effective first-line pharmacologic agent in the management of SVT. Of note, methylxanthines (i.e. caffeine and theophylline) antagonise the effects of adenosine via competitive binding at the purinergic receptor. In addition, adenosine should be avoided in patients with a history of asthma and second or third degree atrioventricular block. In patients receiving carbamazepine, the initial dose of adenosine should be reduced by half due to an increased risk of heart block [3]. Adenosine should always be administered under monitoring of vital signs and a defibrillator should be present on site. If adenosine fails, calcium channel blockers such as verapamil and diltiazem may be administered as an infusion. Like adenosine, non-dihydropyridine calcium channel blockers increase the refractory period and slow atrioventricular nodal conduction and may be used in the acute management of recalcitrant SVT [4].

Newer techniques described in recent literature

An interesting case report published in 1991 described the successful employment of a nasogastric tube to terminate SVT when other vagal manoeuvres had failed [5]. This method worked due to stimulation of the larynx and epiglottis which are areas supplied by the vagus nerve. Over the past few years, several studies have been performed to evaluate the efficacy of the various treatment options available for recalcitrant SVT in the emergency setting. Recent literature has reported the successful use of high dose adenosine (up to $36 \mathrm{mg}$ ) in the acute management of refractory SVT [6]. Calcium channel blockers have been shown to be as efficacious as adenosine in the treatment of SVT and may be useful for refractory AVNRT [7]. Beta blockers have been evaluated but were found to have a lower conversion rate and a higher risk of hypotension compared to calcium channel blockers [8]. Although not specifically described for recalcitrant SVT, modified Valsalva manoeuvres including the upsidedown position [9], as well as forced expiration into a syringe while in the Trendelenburg position have been described to terminate SVT with increased success rates as compared to traditional vagal manoeuvres (43\% vs $17 \%$ ) [10]. Novel techniques such as the use of handstands have also been reported as a simple, non-invasive technique in the treatment of SVT in the paediatric population [11].

Our case is compelling because it describes the occurrence of SVT in two unique contexts: (1) old age and (2) malignancy; the implications of which will be further discussed regarding management of SVT in the emergency department.

Supraventricular tachycardia in the elderly

Although SVT is a tachyarrhythmia which occurs more frequently among younger patients, no age is spared and this arrhythmia is not uncommonly encountered in elderly patients presenting to the emergency department. Although the steps in the acute management of SVT in the elderly are largely similar to that of the younger patient population, there are some notable differences:

1. Unlike the young, elderly patients tend to be more symptomatic and more frequently require emergency treatment including urgent hospital admissions. A study published by Epstein et al. reported that SVT, although well-tolerated in young patients, is more likely to be associated with incapacitating symptoms and may be potentially life-threatening in the elderly [12].

2. Tachycardia tends to be persistent and recurrent in the elderly, which may result in dilated cardiomyopathy and congestive cardiac failure [13].

3. Arrhythmias such as SVT are a considerable cause of falls, disability and frequent admissions to hospital in the elderly [14]. This group of patients are also more likely to present with syncope or presyncope; a high degree of suspicion is required for early recognition and institution of urgent treatment [15].

4. Elderly patients are more vulnerable to the undesired side effects of antiarrhythmic medications, such as orthostatic hypotension, bradycardia, urinary retention, and falls [16]. In addition, antiarrhythmic drugs are less well-tolerated in this group of patients and may be associated with a higher incidence of toxicity [17]. Caution should be undertaken in the prescription of these drugs to elderly patients.

5. With regard to vagal manoeuvres, caution is advised in the consideration of carotid sinus massage in the elderly - even in patients without an audible bruit - due to the risk of carotid atheroembolism and stroke [18].

\section{Supraventricular tachycardia and malignancy}

The most frequently encountered arrhythmia in oncology patients is SVT. Arrhythmias in this group of patients may occur due to a variety of reasons. Causes reported in the literature include primary or secondary cardiac tumours, pericardial constriction, cardiomyopathy secondary to chemotherapy, increased sympathetic states and metabolic derangements. In addition, certain anti-tumour drugs may be potentially arrhythmogenic; examples include anthracyclines, fluorouracil and gemcitabine. SVT occurring in a background of malignancy is often challenging to abort with pharmacological therapy alone. In such situations, synchronised cardioversion must be considered early [19].

\section{Conclusion}

Supraventricular tachycardia is a common occurrence in the emergency department, with affected patients spanning all age groups. Emergency physicians should be familiar with the acute management of supraventricular tachycardia, with special considerations made for the elderly, as well as patients with a known background of malignancy, in whom supraventricular tachycardia may be recalcitrant and less welltolerated. Early recognition of this condition and the institution of urgent treatment is crucial in avoiding potential complications.

\section{References}

1. Helton MR. (2015) Diagnosis and Management of Common Types of Supraventricular Tachycardia. Am Fam Physician. 92(9):793-800.

2. Vagal Maneuvers ACLS com. Acls.com, 2018.

3. UpToDate [Internet]. Uptodate.com. 2018.

4. Prystowsky EN. (1988) The effects of slow channel blockers and beta blockers on atrioventricular nodal conduction. J Clin Pharmacol. 28(1):6-21. 
5. Gupta A, Lennmarken C, Lemming D, Lindqvist L. (1991) Termination of paroxysmal supraventricular tachycardia with a nasogastric tube: a case report. Acta anaesthesiologica scandinavica. 35(8):786-787.

6. Bailey AM, Baum RA, Rose J, Humphries RL. (2016) High-dose adenosine for treatment of refractory supraventricular tachycardia in an emergency department of an academic medical center: a case report and literature review. Journal of Emergency Medicine. 50(3):477-481.

7. Brubaker S, Long B, Koyfman A. (2017) Alternative Treatment Options for Atrioventricular-Nodal-Reentry Tachycardia: an Emergency Medicine Review. The Journal of emergency medicine.

8. R.J. Sung, L. Blanski, J. Kirshenbaum, P. MacCosbe, P. Turlapat y, A.R. (1986) LadduClinical experience with esmolol, a shortacting beta-adrenergic blocker in cardiac arrhythmias and myocardial ischemia. J Clin Pharmacol. 26 (SA); 15-26.

9. Bronzetti G, Brighenti M, Mariucci E, Fabi M, Lanari M, Bonvicini M, Gargiulo G, Pession A. (2018) Upside-down position for the out of hospital management of children with supraventricular tachycardia. International journal of cardiology. 252:106-109.

10. Rezaie S. (2018) The REVERT Trial: A Modified Valsalva Maneuver to Convert SVT - R.E.B.E.L. EM - Emergency Medicine Blog [Internet]. R.E.B.E.L. EM - Emergency Medicine Blog.

11. Hare M, Ramlakhan S. (2014) Handstands: a treatment for supraventricular tachycardia?. Archives of disease in childhood.
12. Epstein LM, Chiesa N, Wong MN, Lee RJ, Griffin JC, Scheinman MM, Lesh MD. (1994) Radiofrequency catheter ablation in the treatment of supraventricular tachycardia in the elderly. Journal of the American College of Cardiology. 23(6):1356-1362.

13. Ablation Therapy of Supraventricular Tachycardia in Elderly [Internet]. Medscape. 2018.

14. O’Mahony D, Foote C. (1998) Prospective evaluation of unexplained syncope, dizziness, and falls among communitydwelling elderly adults. J Gerontol. 53:435-440.

15. Kalusche D, Ott P, Arentz T, Stockinger J, Betz P Roskamm H. (1998) AV nodal re-entry tachycardia in elderly patients: clinical presentation and results of radiofrequency catheter ablation therapy, Coron Artery Dis. 9:359-363.

16. Lee HC, Huang KT, Shen WK. (2011) Use of antiarrhythmic drugs in elderly patients. Journal of geriatric cardiology: JGC. 8(3):184.

17. Brembilla-Perrot B, Sellal JM, Olivier A, Villemin T, Beurrier D, Vincent J, Manenti V, De Chillou C, Bozec E, Girerd N. (2018) Influence of advancing age on clinical presentation, treatment efficacy and safety, and long-term outcome of inducible paroxysmal supraventricular tachycardia without pre-excitation syndromes: A cohort study of 1960 patients included over 25 years. PloS one. 13(1):0187895.

18. Sohinki D, Obel OA. (2014) Current trends in supraventricular tachycardia management. The Ochsner Journal. 14(4):586-595.

19. Yeung S, Escalante C. (2018) Circulatory Oncologic Emergencies.

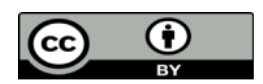

This work is licensed under Creative Commons Attribution 4.0 License

\section{To Submit Your Article Click Here: Submit Manuscript}

DOI: $10.31579 / 2690-4861 / 115$
Ready to submit your research? Choose Auctores and benefit from:

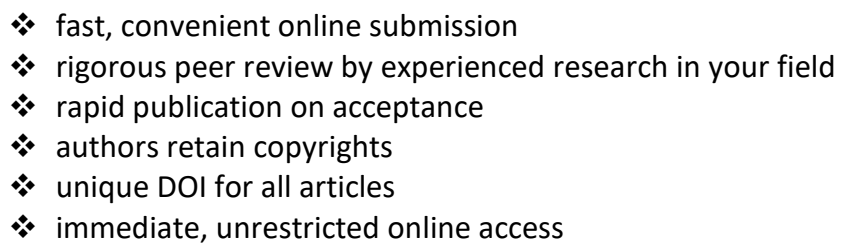

At Auctores, research is always in progress.

Learn more www.auctoresonline.org/journals/international-journal-ofclinical-case-reports-and-reviews 